\title{
FIRST $L$-BAND INTERFEROMETRIC OBSERVATIONS OF A YOUNG STELLAR OBJECT: PROBING THE CIRCUMSTELLAR ENVIRONMENT OF MWC 419
}

\author{
S. Ragland ${ }^{1}$, R. L. Akeson ${ }^{2}$, T. Armandroff ${ }^{1}$, M. M. Colavita ${ }^{3}$, W. C. Danchi ${ }^{4}$, L. A. Hillenbrand ${ }^{5}$, R. Millan-Gabet ${ }^{2}$, \\ S. T. Ridgway ${ }^{6}$, W. A. Traub ${ }^{3}$, G. Vasisht ${ }^{3}$, and P. L. Wizinowich ${ }^{1}$ \\ ${ }^{1}$ W. M. Keck Observatory, 65-1120 Mamalahoa Hwy, Kamuela, HI 96743, USA; sragland@keck.hawaii.edu \\ ${ }^{2}$ NExScI, California Institute of Technology, 770 South Wilson Avenue, Pasadena, CA 91125, USA \\ 3 Jet Propulsion Laboratory, California Institute of Technology, M/S 301-451, 4800 Oak Grove Dr., Pasadena CA, 91109, USA \\ ${ }^{4}$ NASA Goddard Space Flight Center, Exoplanets and Stellar Astrophysics, Code 667, Greenbelt, MD 20771, USA \\ 5 California Institute of Technology, Pasadena, CA 91125, USA \\ ${ }^{6}$ National Optical Astronomy Observatories, P.O. Box 26732, Tucson, AZ 85726-6732, USA \\ Received 2009 April 9; accepted 2009 July 20; published 2009 August 25
}

\begin{abstract}
We present spatially resolved $K$ - and $L$-band spectra (at spectral resolution $R=230$ and $R=60$, respectively) of MWC 419, a Herbig Ae/Be star. The data were obtained simultaneously with a new configuration of the $85 \mathrm{~m}$ baseline Keck Interferometer. Our observations are sensitive to the radial distribution of temperature in the inner region of the disk of MWC 419. We fit the visibility data with both simple geometric and more physical disk models. The geometric models (uniform disk and Gaussian) show that the apparent size increases linearly with wavelength in the $2-4 \mu \mathrm{m}$ wavelength region, suggesting that the disk is extended with a temperature gradient. A model having a power-law temperature gradient with radius simultaneously fits our interferometric measurements and the spectral energy distribution data from the literature. The slope of the power law is close to that expected from an optically thick disk. Our spectrally dispersed interferometric measurements include the $\mathrm{Br} \gamma$ emission line. The measured disk size at and around $\mathrm{Br} \gamma$ suggests that emitting hydrogen gas is located inside (or within the inner regions) of the dust disk.
\end{abstract}

Key words: circumstellar matter - instrumentation: interferometers - stars: emission-line, Be - stars: individual (MWC 419) - stars: pre-main sequence - techniques: interferometric

\section{INTRODUCTION}

Pre-main sequence (PMS) stars fall to the upper right of the main sequence on the Hertzsprung-Russell (H-R) diagram, journeying toward the main sequence via radial contraction. For the first several million years, a PMS star is surrounded by a disk of gas and dust left over from the early stage of star formation.

The evolution of a disk is not well understood. Circumstellar disks provide the raw material for planet formation. Thus, understanding the evolution of a disk helps us to understand planet formation. Clues to the physical conditions of planet formation and hence future suitability for life on planets other than Earth potentially can be inferred from a detailed characterization of inner young stellar object (YSO) disks.

Herbig $\mathrm{Ae} / \mathrm{Be}(\mathrm{HAeBe})$ stars are intermediate-mass PMS stars with spectral types earlier than G0, located close to the zero-age main sequence. Early spectral energy distribution (SED) modeling efforts (Hillenbrand et al. 1992) to explain the infrared excess of $\mathrm{HAeBe}$ stars assumed (1) optically thick but geometrically thin circumstellar disks with inner optically thin holes, up to several stellar radii, in order to account for the observed inflections in the $1-5 \mu \mathrm{m}$ region, and (2) relatively large accretion rates, on the order of $10^{-6} M_{\odot} \mathrm{yr}^{-1}$, in order to account for the strength of the $3.8 \mu \mathrm{m}$ ( $L$-band) excess. It was quickly pointed out (Hartmann et al. 1993) that at such a large mass accretion rate, the gas in the inner dust-free hole is expected to be optically thick and to produce excess nearinfrared emission, which is inconsistent with the dip seen in the near-infrared SED. For the relatively low accretion rates later inferred for $\mathrm{HAeBe}$ objects, disk models could not explain the observed SEDs in the near-infrared region. Puffed-up inner dust rim models were introduced (Natta et al. 2001) and later refined (Dullemond et al. 2001; Isella \& Natta 2005) to ameliorate the shortcomings of the classical disk models. These later models attribute the near-infrared excess to stellar radiation shining directly on the inner dust edge of the dust disk. Tuthill et al. (2001) directly imaged the high luminosity YSO LkH $\alpha 101$ and independently proposed that the bulk of the near-infrared emission arises in a hot ring located at the dust sublimation radius.

Long-baseline interferometric observations provide the milliarcsecond angular resolution required to resolve the planetforming structures immediately surrounding PMS stars. Such high-angular-resolution infrared observations are well suited for probing near-circumstellar environments since the inner zones of the circumstellar disks and envelopes emit primarily at nearinfrared wavelengths. A large number of YSOs across the luminosity range have now been spatially resolved at near-infrared wavelengths (Millan-Gabet et al. 2001, 2007; Colavita et al. 2003; Eisner et al. 2003, 2004, 2007, 2009; Monnier et al. 2005; Akeson et al. 2005; Isella et al. 2008; Tannirkulam et al. 2008), showing that indeed the characteristic sizes correlate strongly with central luminosity, lending support to the "puffed-up" inner dust rim paradigm, especially for Herbig Ae objects. However, it was also pointed out (Monnier \& Millan-Gabet 2002; Eisner et al. 2004; Monnier et al. 2005) that most higher luminosity Herbig Be objects are considerably undersized compared with the predictions of this model, and in better agreement with the "classical" models featuring optically thick emission that extends inward very close to the central star.

MWC 419 is a photometrically variable emission-line star that is in many ways typical of the $\mathrm{HAeBe}$ class, including illumination of a reflection nebula (Herbig 1960). MWC 419 has spectral type B8 (Herbig 1960), luminosity $330 L_{\odot}$ (Hillenbrand et al. 1992), and distance $650 \mathrm{pc}$ (Hillenbrand et al. 1992); it is also known as V594 Cas or BD+61 154. Narrow band $\mathrm{H} \alpha$ imaging 
studies show unipolar large-scale structures around MWC 419 (Marston \& McCollum 2008). Interestingly, these images show two lobes on the southeast side of MWC 419-the outer lobe extending to $\sim 4$ arcmin-suggesting a history of episodic mass loss in this PMS star's evolution. These authors attribute the nondetection of northwest lobes to obscuration. These large-scale structures have no influence on our observations of the central region. The measured intrinsic polarization of MWC 419 in the $V$-band is $0.53 \%$ (Hillenbrand et al. 1992), suggesting that the contributions from reflected optical light to the total emission is insignificant. No close companions to MWC 419 are known (Pirzkal et al. 1997; Bernacca et al. 1993). The star has P-Cygni characteristics in the lower Balmer lines and the implied wind has been modeled in detail by Bouret \& Catala (1998). However, it has also been considered as a $\mathrm{B}[\mathrm{e}]$ star and interpreted as undergoing post-main sequence evolution rather than pre-main sequence evolution. The star is projected on the outer regions of the young association NGC 225 (Herbig 1960; Subramaniam et al. 2006), within a small area of enhanced extinction and CO emission (Hillenbrand 1995; Fuente et al. 2002) that is associated with the dark cloud L 1302 (van den Ancker et al. 1998). However, the membership of the star in the cluster is debated (Lattanzi et al. 1991). The age of the cluster is often quoted as 120 Myr (Lattanzi et al. 1991) but Subramaniam et al. (2006) have argued based on the presence of a number of young stars that it is really a $<10$ Myr pre-main sequence population.

As an IRAS (Infrared Astronomical Satellite) and ISO (Infrared Space Observatory) source, the infrared dust spectrum and the SED of MWC 419 have been discussed by several authors (Brooke et al. 1993; Chen et al. 2000; Berrilli et al. 1992; Hillenbrand et al. 1992; Miroshnichenko et al. 1997; Pezzuto et al. 1997). No Spitzer data were obtained for this source, but it has a weak silicate emission at $10 \mu \mathrm{m}$ in groundbased data (Chen et al. 2000).

MWC 419 was previously observed interferometrically using the Palomar Testbed Interferometer (PTI) and was spatially resolved in the $K$-band with a reported uniform-disk angular diameter of $3.34 \pm 0.16$ milliarcsec (mas) or a Gaussian distribution FWHM of $2.07 \pm 0.11$ mas (Wilkin \& Akeson 2003). MWC 419 was not resolved from the Infrared Optical Telescope Array (IOTA) interferometer observations (MillanGabet et al. 2001), where the angular resolution was $\sim 6$ mas at $2.2 \mu \mathrm{m}$.

The Keck Interferometer (KI), with $\sim 3$ mas angular resolution at $2.2 \mu \mathrm{m}$, can resolve the inner disks ( $\sim 1 \mathrm{AU})$ of nearby PMS stars. In this paper, we report observations of MWC 419 using multicolor interferometry at well-separated wavelengths, here $K$-band (2.0-2.4 $\mu \mathrm{m})$ and $L$-band (3.5-4.1 $\mu \mathrm{m})$. The simultaneous $K$ - and $L$-band interferometric measurements enable us to probe different regions ( $\sim 1300 \mathrm{~K}$ and $800 \mathrm{~K}$ respectively) of the inner circumstellar disks of PMS stars. Discrete spatial distributions, such as dust rims, and relatively smooth spatial distributions, such as classical accretion disks, are expected to have different size versus wavelength behaviors and can be distinguished in such multicolor observations at well-separated wavelengths. In addition, interferometric measurements in the relatively unexplored $L$-band provide further constraints to the disk/ envelope geometry via temperature- and density-sensitivity of different models. Multicolor interferometry at well-separated wavelengths ( $H, K$ and $N$-bands) was recently reported for two other YSO disks, namely, MWC 297 (Acke et al. 2008) and MWC 147 (Kraus et al. 2008a) from Very Large Telescope Interferometer (VLTI) observations.
In Section 2, we present our observations and data reduction. In Section 3, we describe the data analysis where we fit the visibility-squared $\left(V^{2}\right)$ data with various YSO disk models. In Section 4, we discuss our results and in Section 5 we provide a brief summary.

\section{OBSERVATIONS AND DATA REDUCTION}

$\mathrm{KI}$ is a near- and mid-infrared long-baseline interferometer consisting of two $10 \mathrm{~m}$ diameter apertures separated by a $B=85$ $\mathrm{m}$ baseline at a position angle of $\sim 38^{\circ}$ east of north. Both Keck telescopes are equipped with adaptive optics. The maximum resolution of our KI observations is $\lambda / 2 B \sim 2.7$ mas and $\sim 4.5$ mas in the $K$-band and $L$-band, respectively.

The $L$-band science instrument, consisting of an infrared camera and beam-combiner optics, is similar to the existing $H$ - and $K$-band fringe tracker (FATCAT) routinely used for $V^{2}$ measurements since 2002 (Colavita et al. 2003). We obtained first light with this new instrument in 2008 April. The $L$-band limiting magnitude of 6 is set by the requirement for a broadband phase signal-to-noise ratio $(\mathrm{S} / \mathrm{N})$ of 10 in the $10 \mathrm{~ms}$ fringetracker frame. The total integration time on source is typically $200 \mathrm{sec}$, which provides good $\mathrm{S} / \mathrm{N}$ for $V^{2}$ measurements in the 10 spectral channels.

The $L$-band instrument uses a PICNIC focal plane array detector with a $5 \mu \mathrm{m}$ cutoff wavelength, while FATCAT uses a HAWAII array. The detector electronics are similar for both instruments. Details of this $L$-band instrument are given by Ragland et al. (2008).

For the current work, a prototype multiwavelength observing capability was used, enabling simultaneous $K$ - and $L$-band observations of our science target. In this configuration, the telescope pupil is split into left and right-halves at the dual star modules of both telescopes and routed through separate coude paths utilizing the existing beam-train infrastructure for the nulling mode. The left-half pupils of both telescopes are combined by the $K$-band instrument and the right-half pupils are combined by the $L$-band instrument.

The $K$ - and $L$-band science instruments each have two complementary interferometric outputs with different spectrometers on each. For the $L$-band, these are a (pseudo) broad band and a low-dispersed mode (10 channels across the $L$-band; $R=60$ ). For the $K$ band, these are a broad band and a medium dispersion mode (42 channels across the $K$-band; $R=230$ ).

The field of view of the instrument, defined by the singlemode fibers that couple that light to the detector array, is $\sim 55$ mas for the $K$-band and $\sim 93$ mas for the $L$-band observations. These field restrictions were enforced in our modeling work.

The ZABCD algorithm (Colavita et al. 1999), defined as below, is used for fringe tracking and science measurements. In this procedure, five reads are made while the fast delay line scans over one wavelength $(2.2 \mu \mathrm{m}$ for the $K$-band and $3.7 \mu \mathrm{m}$ for the $L$-band); explicitly, the reads are the reset pedestal $(z)$, followed by four nondestructive reads $(\mathrm{a}, \mathrm{b}, \mathrm{c}, \mathrm{d})$ spaced at $\lambda / 4$ intervals as the detector integrates up. For each quarter wave bin, we then calculate $A=a-z, B=b-a$, etc. These values are used to estimate the square of the fringe visibility $\left(V^{2}\right)$ for science and the fringe phase and $\mathrm{S} / \mathrm{N}$ for fringe tracking. We apply corrections to the bin data to account for detector bias and for differences between the length of the delay scan and the actual wavelength. Residual instrument bias and atmospheric seeing effects are corrected by observing a calibrator of known $V^{2}$ under similar observing conditions. Essentially, we estimate instrument transfer function using the bracketing observations of 
calibrator stars with known angular diameters using a weighted averaging scheme that considers the time and sky proximity of calibrators relative to the target. ${ }^{7}$

The observations reported here were taken on the night of UT 2008 August 19. We observed two calibrators-HD 1843 and HD 6210-under similar observing conditions as the science target to calibrate the science data. The adopted angular sizes of the calibrators are $1.0 \pm 0.02$ and $0.5 \pm 0.01$ mas, respectively (van Belle 1999). We performed bracketed calibration meaning that our observing sequence included calibrator measurements before and after each target.

The measurements presented here were taken over a narrow range of position angles $\left(25^{\circ}-33^{\circ}\right)$ and projected baselines (76.1-77.8 $\mathrm{m}$ ) and, hence, no attempt was made to derive an inclination angle and position angle of the disk. For the purpose of this article, we assume that the disk is face-on. Thus, the sizes reported here are the values along the position angle of about $29^{\circ}$, and the actual size of the disk could be larger depending on the inclination angle and the position angle. Our $K$-band measurements, taken at the position angle of about $29^{\circ}$, gives similar disk size as that of previous PTI observations, taken at the position angle of about $83^{\circ}$. Hence, a face-on disk for MWC 419 is a reasonable assumption in the absence of necessary high-spatial resolution observations.

\section{ANALYSIS}

In this section, we fit our measurements with different models of increasing complexity and increasing physical realism.

The measured $V^{2}$ includes contributions from the central star $\left(V_{*}^{2}\right)$ and the bright circumstellar disk. The visibility square $\left(V_{\text {disk }}^{2}\right)$ of the circumstellar disk of MWC 419 is obtained from the measured data by removing the contributions from the central star (Millan-Gabet et al. 2001) using

$$
V_{\text {measured }}^{2}=\left(\frac{F_{*} V_{*}+F_{\text {disk }} V_{\text {disk }}}{F_{*}+F_{\text {disk }}}\right)^{2} .
$$

The adopted disk-to-star flux ratio $\left(F_{\text {disk }} / F_{*}\right)$ is 12 and 40 in $K$ and $L$ based on our SED analysis discussed in Section 3.3. The central star is assumed to be unresolved for our observations (i.e., $V_{*}=1.0$ ), which is a reasonable assumption for a $\mathrm{B} 8 \mathrm{star}$ at a distance of $650 \mathrm{pc}$. These corrections yield values of $V_{\text {disk }}^{2}$ that are smaller than the total $V^{2}$ by $0.04(\sim 9 \%)$ and $0.01(\sim 2 \%)$ in $K$ and $L$, respectively. The $K$-band $V^{2}$ measurements are consistent with earlier $K$ broadband measurements and provide additional spectrally resolved information within the $K$-band spectral region.

The visibilities of the disk models (Sections 3.2 and 3.3) are computed by numerically summing the contributions from annular rings of infinitesimally small widths and weighting them by their respective flux contributions; for this purpose, we divided the disk radially into 5000 annular rings, in logarithmic scale. The normalized visibility for a uniformly bright annular ring with an inner diameter of $\theta_{i n}$, an outer diameter of $\theta_{\text {out }}$ and $\theta_{\text {in }} \lesssim \theta \lesssim \theta_{\text {out }}$, can be written as

$$
\begin{aligned}
V_{\mathrm{ann}}(B, \lambda, \theta)= & \left(\frac{2 \lambda}{\pi B\left(\theta_{\text {out }}^{2}-\theta_{\text {in }}^{2}\right)}\right)\left(\theta_{\text {out }} J_{1}\left(\pi B \theta_{\text {out }} / \lambda\right)\right. \\
& \left.-\theta_{\text {in }} J_{1}\left(\pi B \theta_{\text {in }} / \lambda\right)\right),
\end{aligned}
$$

where $J_{1}$ is the first-order Bessel function, $B$ is the projected baseline, and $\lambda$ is the wavelength of observation. The flux from such an annular uniform ring can be written as

$$
F_{\text {ann }}(\lambda, \theta)=\frac{\pi}{4} P(T, \lambda)\left(\theta_{\text {out }}^{2}-\theta_{\text {in }}^{2}\right),
$$

where $P(T, \lambda)$ is the Planck blackbody function and $T$ is the mean temperature of the annular ring.

Now, the visibility of the total disk can be written as

$$
V_{\mathrm{disk}}(B, \lambda)=\sum\left(V_{\mathrm{ann}}(B, \lambda, \theta) \times \frac{F_{\mathrm{ann}}(\lambda, \theta)}{\sum F_{\mathrm{ann}}(\lambda, \theta)}\right),
$$

where $\sum F_{\text {ann }}(\lambda, \theta)$ is the total disk flux derived by summing all the annular rings of the disk in order to normalize the flux. We adopted a nonlinear least-squares fitting method to minimize the chi-square for all our modeling work.

For our SED analysis, photometric measurements from the literature are used, after interstellar extinction corrections using the extinction law of Cardelli et al. (1989). The extinction for MWC 419 in the $V$-band is assumed to be $A_{v}=2.1$ (Hillenbrand et al. 1992).

The models presented in this article assume face-on geometry for the disk. A disk with an inclination angle $\phi$ could predict a larger inner disk radius depending on the inclination angle and the position angle of the projected interferometer baseline on the sky. In this case, SED models would underestimate the flux at all wavelengths by a factor of $\cos (\phi)$. In other words, classical accretion disk models would have a factor of $\cos (\phi)$ larger accretion rate to explain the SED data and power-law models would have a relatively larger disk temperature at all radial distances.

\subsection{Simple Wavelength-dependent Geometrical Models}

The measurements presented in this article consist of spectrally dispersed data within the $K$ - and $L$-bands. As stated above, the $K$ and $L$ visibilities corrected for the stellar contribution are different, suggesting a wavelength dependence to the size of the spatially resolved emission. We can use the spectrally dispersed data to investigate in more detail this wavelength dependency. We choose three geometrical models, namely, uniform disk, Gaussian distribution, and ring models, to fit to our measurements.

The complex visibilities for a normalized pole-on uniform disk and a Gaussian distribution can be written as follows:

$$
\begin{gathered}
V_{U D}(B, \lambda)=\frac{2 J_{1}\left[\pi B \theta_{U D} / \lambda\right]}{\pi B \theta_{U D} / \lambda}, \\
V_{\text {Gaussian }}(B, \lambda)=\exp \left(-\frac{\left(\pi B \theta_{\mathrm{FWHM}} / \lambda\right)^{2}}{4 \ln 2}\right) .
\end{gathered}
$$

Here $\theta_{U D}$ is the uniform-disk angular diameter of the circumstellar disk, $\theta_{\text {FWHM }}$ is the full-width at half maximum (FWHM) of the Gaussian distribution, and other terms as above. See Equation (2) for the ring model.

To start, we fit the data with a wavelength-independent geometrical model (Figure 1). The derived uniform disk, Gaussian, and ring angular sizes, from simultaneous fits to $K$ and $L$ band data, are 3.59 \pm 0.01 mas, $2.21 \pm 0.01$ mas (FWHM), and 3.91 \pm 0.01 mas, respectively. The corresponding reduced-chi-square $\left(\chi_{R}^{2}\right)$ values are $36.1,35.0$, and 38.3, respectively. As indicated by the very poor $\chi_{R}^{2}$ values, the uniform disk, Gaussian, and ring

\footnotetext{
http://nexsci.caltech.edu/software/V2calib/
} 


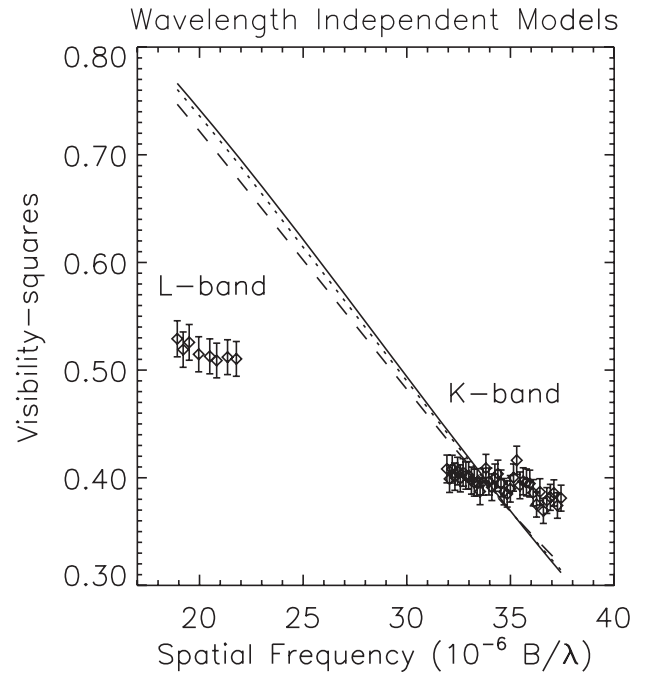

Figure 1. Wavelength-independent model fits. The multiwavelength measurements (diamond symbols) are plotted against spatial frequency. Also shown are geometrical model fits to the data. The dotted-line, dashed-line, and continuous line refer to uniform disk, Gaussian, and ring models, respectively. The poor model fits indicate that these simple models are an inadequate description of the data.

models fit neither the $K$ and $L$ data in the mean nor the slope of the visibilities throughout either the $K$ - or $L$-bands.

As the wavelength-independent size models fail to fit the measurements, we fit the data with wavelength-dependent sizes. The results of these geometrical model fits to the measured data points are shown in Figure 2.

The observed wavelength dependence in the $2-4 \mu \mathrm{m}$ region has a simple linear relationship to first order. The parameters of a linear fit to the derived apparent wavelength dependent diameters are given in Figure 2. The $\chi_{R}^{2}$ value for this model fit is 2.1. The measured uniform-disk diameter in the center of the $L$-band $\left(\theta_{L}=5.04\right.$ mas) is $\sim 44 \%$ larger than in the center of the $K$-band ( $\theta_{K}=3.49$ mas). The steep slopes of these linear relations suggest that the $2-4 \mu \mathrm{m}$ emission source must be extended with strong radial temperature dependence. Earlier multicolor (near- and mid-infrared) interferometry of two YSO disks has shown similar wavelength dependence (Acke et al. 2008; Kraus et al. 2008a). In the following section, we explore more complex extended disk models to explain our observations.

\subsection{Geometrically Thin, Optically Thick Accretion Disk Models}

We compared our interferometric data with an accretion disk model of Hillenbrand et al. (1992) based on an SED analysis. In this classical accretion disk model, the temperature distribution of dust is derived by combining the contributions from a radiatively heated reprocessing component $T_{\text {rep }}$ and a viscously heated accretion component $T_{\text {acc }}$. The parameters of the disk are the following: The inner disk (hole) radius is $R_{i n}=$ $0.22 \mathrm{AU}\left(10 R_{*}\right)$, the outer disk radius is $62 \mathrm{AU}$, and the mass accretion rate $(M)$ is $1.98 \times 10^{-5} M_{\odot} \mathrm{yr}^{-1}$. The stellar radius is $4.8 R_{\odot}$, the stellar effective temperature is $11,220 \mathrm{~K}$, the distance to the star is $650 \mathrm{pc}$, and the stellar mass is $5.3 M_{\odot}$. The inner disk temperature is $2480 \mathrm{~K}$ and the outer disk temperature is $40 \mathrm{~K}$. The resulting flat accretion disk model highly overestimates $V^{2}$ in the $K$ - and $L$-bands. We get a reduced-chi-square $\left(\chi_{R}^{2}\right)$ value of 664 for the interferometric data. The model results are given in Table 1 . The physical reason that the classical accretion disk model fails is that it creates too much flux in the inner region of the disk because of the added accretion luminosity, making the angular size much smaller than observed.

We have also fit visibility data with a similar accretion disk model (Figure 3) by treating the inner disk (hole) radius as a free model parameter. In addition, the mass accretion rate was increased by a factor of 1.5 (i.e., $2.97 \times 10^{-5} M_{\odot} \mathrm{yr}^{-1}$ ) in order to fit SED data. The $\chi_{R}^{2}$ value for this model fit to interferometric measurements is 2.59. The derived inner disk (hole) radius is $1.66 \pm 0.01$ mas $\left(0.54 \mathrm{AU} ; \sim 24 R_{*}\right)$ and the inner disk temperature is $1457 \mathrm{~K}$. The outer disk radius is fixed at $50 \mathrm{AU}$, where the disk temperature is $51 \mathrm{~K}$. This model fits the $K$ band data well, but is about $3 \sigma$ too high in $V^{2}$ compared with the $L$ band data. The extremely large accretion luminosity required by the SED data poses challenges to these two accretion disk models. Thus, the classical model that could fit the SED fails here, just like in the previous interferometric studies of Herbig Ae/Be disks (Millan-Gabet et al. 2001; Eisner et al. 2004). Moreover, it is not sufficient to adjust the inner radius and temperature of the disk. A modification of the disk temperature profile is needed as in the case of more recent studies (Eisner et al. 2007; Acke et al. 2008; Kraus et al. 2008a); this is discussed in the following section.

\subsection{Geometrically Thin, Optically Thick Disk with Power-Law Temperature Distribution}

The wavelength dependence of the measured visibilities and SED data shown above implies an inverse $T(r)$ relationship. We fit our data with a simple model with a power-law temperature gradient of the functional form $T(r) \propto r^{-\alpha}$, where $r$ is the radial distance from the central star and $\alpha$ is the power-law parameter. The model $V^{2}$ is derived using Equations (2) and (3) with a power-law temperature distribution. The radius of the inner disk and $\alpha$ are treated as free parameters. The temperature
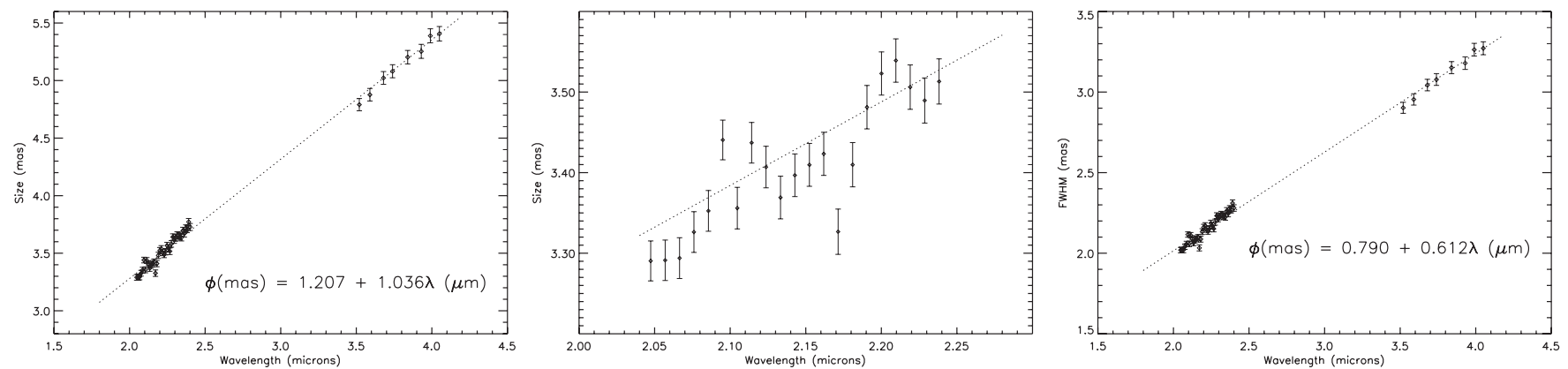

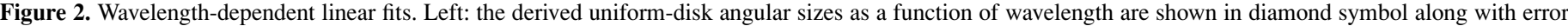

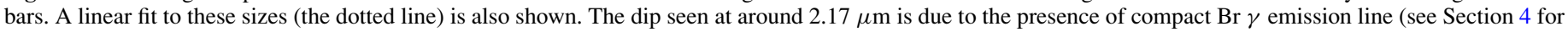
details). Middle: same as the left figure, but a zoomed view of the $\mathrm{Br} \gamma$ emission-line region. Right: same as the left figure except for a Gaussian distribution. 

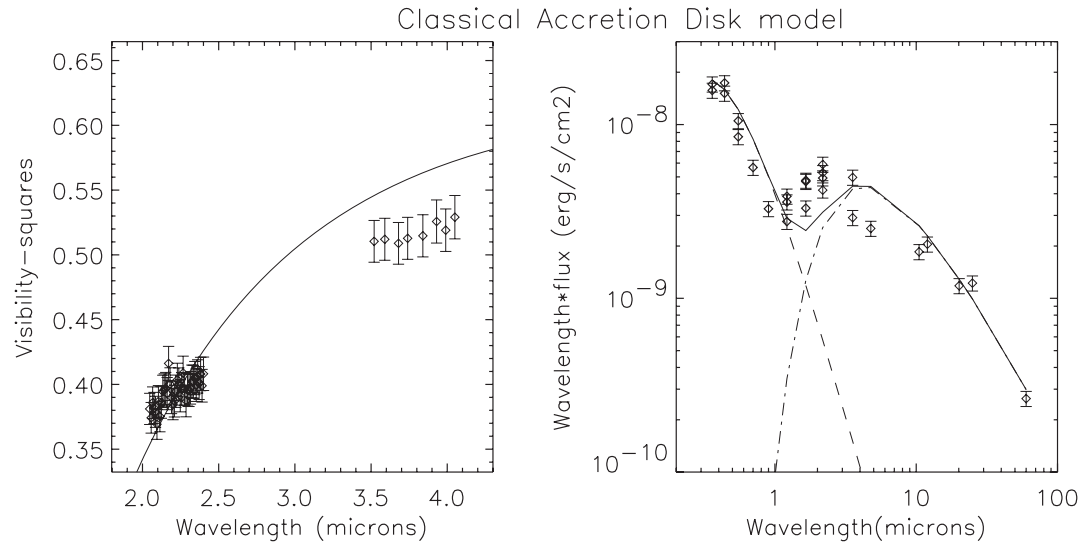

Figure 3. Classical accretion disk model fits. Interferometric data points are shown with error bars. Shown in continuous line is an accretion disk model with the inner radius of the disk (hole size) treated as a free model parameter. The outer radius of the disk is fixed at 50 AU. Right: photometric data taken from the literature are shown along with the SED model for the same accretion disk model. The dashed line shows the blackbody spectral distribution of the central star and the dashed-dotted line shows the blackbody emission from the disk.
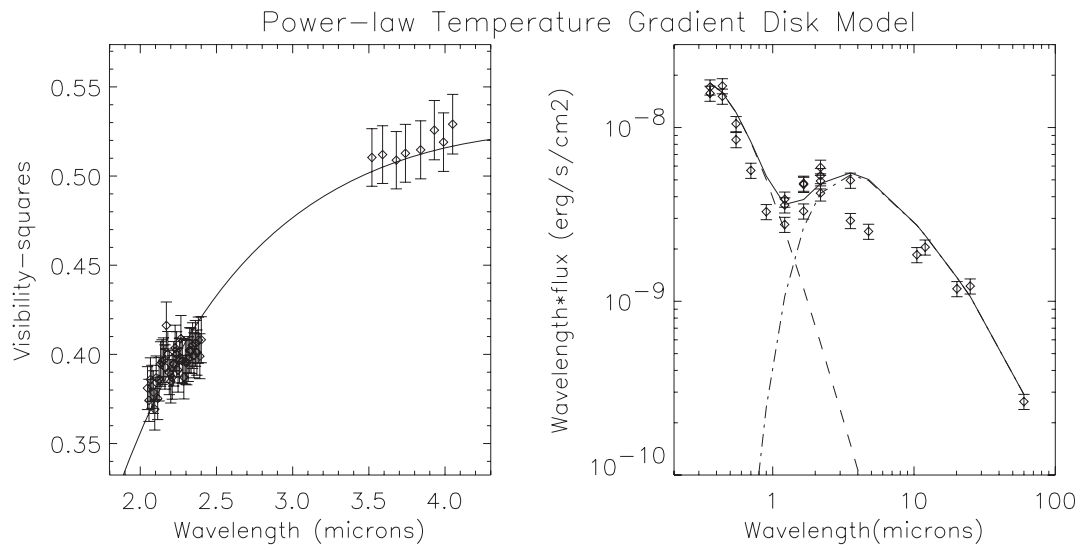

Figure 4. Power-law disk model fits. Left: interferometric data points are shown with error bars. The solid line is a power-law temperature gradient disk model. The power-law parameter, the inner disk radius, and the inner disk temperature are free parameters. The outer radius is fixed at 20 AU. Right: photometric data taken from the literature are shown with error bars. The solid line is the SED model for the same accretion disk model. The dashed and dashed-dotted lines are the SEDs of the star and the disk.

Table 1

Derived Parameters for the Disks Models Presented in Sections 3.2 and 3.3

\begin{tabular}{|c|c|c|c|c|c|}
\hline Model & $\begin{array}{l}R_{\text {in }} \\
(\mathrm{AU})\end{array}$ & $\begin{array}{l}T_{\text {in }} \\
(\mathrm{K})\end{array}$ & $\alpha$ & $\begin{array}{c}\dot{M} \\
\left(\times 10^{-5} M_{\odot} \mathrm{yr}^{-1}\right)\end{array}$ & $\chi_{R}^{2}$ \\
\hline $\begin{array}{l}\text { Classical accretion disk } \\
\text { (parameters fixed at published values) }\end{array}$ & 0.22 & 2480 & 0.75 & 1.98 & 664 \\
\hline $\begin{array}{l}\text { Classical accretion disk } \\
\left(R_{\text {in }} \text { and } \dot{M} \text { varied }\right)\end{array}$ & $0.538 \pm 0.001$ & 1457 & 0.75 & 2.97 & 2.59 \\
\hline Modified power-law disk & $0.477 \pm 0.005$ & 1800 & $0.71 \pm 0.01$ & $\cdots$ & 0.73 \\
\hline
\end{tabular}

Note. The $\chi_{R}^{2}$ values are for the interferometer data (only).

of the inner disk is fixed at $1800 \mathrm{~K}$ and the optical depth at 1 for the entire disk in order to satisfy the SED data; a larger value for the optical depth overestimates the disk flux. The resultant model fits are shown in Figure 4. The derived inner disk radius is $1.47 \pm 0.02$ mas $\left(0.477 \mathrm{AU} ; \sim 21 R_{*}\right)$ and the derived value for $\alpha$ is $0.71 \pm 0.01$. The outer radius is fixed at $20 \mathrm{AU}$ (in order for the SED to be consistent with the far-infrared photometric data) and the corresponding temperature is $125 \mathrm{~K}$. The $\chi_{R}^{2}$ for this model fit to our interferometric data is 0.73 and the sensitivity of $\chi_{R}^{2}$ to the model parameters is shown in Figure 5. As the powerlaw disk model is a satisfactory fit, we consider it the simplest model that explains the data. However, one can also conceive of more complex and physically plausible models such as optically thin inner disk regions (holes) surrounded by optically thick outer disks, perhaps including puffed-up inner rims. Our initial exploration of such models suggests that they can also fit our data, but the interferometric data themselves do not drive us to such complex models.

\subsection{Binary Star Model}

In this section, we investigate whether the data can be explained, without invoking hot/warm circumstellar dust, by the effect of a companion star. The visibility model for a binary system can be written as

$$
V_{\text {binary }}(\lambda)=\frac{\sqrt{V_{p}^{2}+R^{2} V_{s}^{2}+2\left|V_{p}\right|\left|V_{s}\right| R \cos ((2 \pi / \lambda) \mathbf{B} \cdot \mathbf{s})}}{1+R},
$$




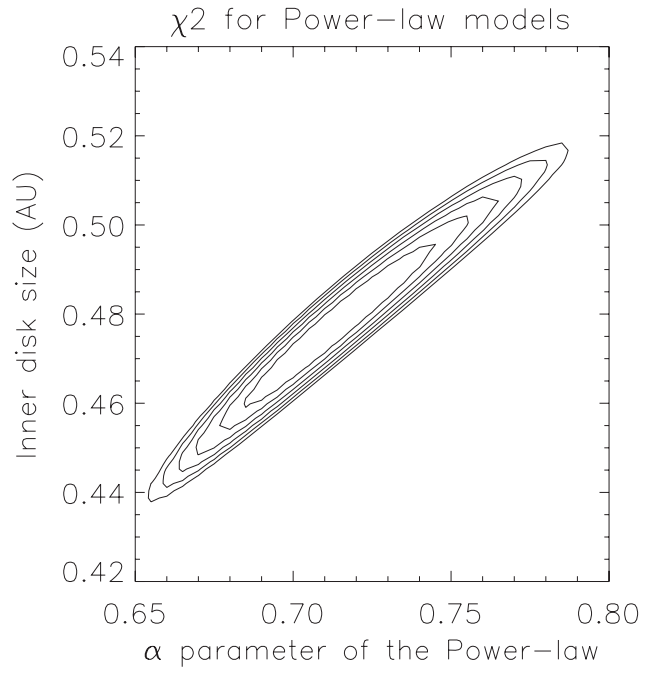

Figure 5. Contour map of $\chi_{R}^{2}$ as a function of the power-law parameter $\alpha$ and the inner disk radius. The contour lines-from inner to the outer-refer to $\chi_{R}^{2}$ values of $1.0,1.2,1.4,1.6,1.8$, and 2.0

where $R$ is the secondary-to-primary flux ratio, $V_{p}$ and $V_{s}$ are visibilities of primary and secondary components, and $\mathbf{s}$ is the binary separation.

The derived parameters from a binary model fit to our measurements are the following: projected binary separation along the position angle of $29^{\circ}$ (east of north) $=5.12 \pm 0.01$ mas, primary-to-secondary flux ratio $=11.79 \pm 0.30$, and uniformdisk angular diameter of the primary component (YSO disk size $)=3.53 \pm 0.01$ mas (a binary model with two point sources fails to fit SED data). The diameter of the secondary component is fixed at 0.001 mas (effectively unresolved). The model fit to the data is shown in Figure 6. The $\chi_{R}^{2}$ value is 0.94 . In this scenario, the secondary component would have the same $K-L$ color of 1.34 as the primary YSO disk, suggesting that the potential companion were a YSO disk with significant IR excess. In this case, our observations would have resolved the disk around the companion. Hence, we also fit our measurements by fixing the companion size to 2 mas in order to explore this possibility. The derived parameters for this binary model are the following: projected binary separation along the position angle of $29^{\circ}$ (east of north) $=5.23 \pm 0.01$ mas, primary-tosecondary flux ratio $=11.6 \pm 0.3$, and uniform-disk angular diameter of the primary component $($ YSO disk size $)=3.51 \pm$
0.01 mas. The model fit to the data is also shown in Figure 6. The $\chi_{R}^{2}$ value is 1.57 , but additional observations at other baseline orientations would provide significant additional constraints on the binary model. The derived flux ratio of $\sim 12$ suggests a cooler stellar companion with a smaller inner disk radius compared with the primary star. However, such a companion model would overestimate SED at the shorter wavelengths because of the flux from the companion photosphere. Hence, a binary scenario is not favored as an explanation for our measurements.

\section{DISCUSSION}

The KI measurements presented here agree with previous observations from PTI of the $K$-band size of MWC 419 (Wilkin $\&$ Akeson 2003). The addition of the spectrally dispersed $K$ band information and new $L$-band observations, also spectrally dispersed, provides powerful new constraints on the physical structure of the material surrounding the central star. As shown in the leftmost panel of Figure 2, the wavelength dependence of the size is apparent within both the $K$ and $L$ bands; the combination of the two bands allows an even tighter constraint on the temperature power-law than possible using either data set alone. The radial temperature profiles of the disk models presented in Sections 3.2 and 3.3 are shown in Figure 7. The temperature profile of the best-fit power-law model is very similar to that of a classical, geometrically thin accretion/ reprocessing disk $(\alpha=-0.75)$.

As discussed earlier (Section 3.2), the accretion disk model requires an unrealistically large accretion rate, on the order of a few times $10^{-5} M_{\odot} \mathrm{yr}^{-1}$ in order to fit the SED data. Simple estimates of the accretion rate for this star range from $5 \times 10^{-7}$ to $10^{-6} M_{\odot} \mathrm{yr}^{-1}$ based on mass-loss data and upper limits to radio emission at $3.6 \mathrm{~cm}$ (Kurchakov et al. 2007; Boehm \& Catala 1995; Skinner et al. 1993). The necessity of an unrealistically large accretion rate in the accretion disk model is probably an artifact of the physically unrealistic assumption of a zerothickness disk that causes the reprocessing of radiation to be very inefficient. Hence, we do not favor an accretion disk model for the MWC 419 disk.

A power-law temperature profile with a radius in which the radial exponent is a free parameter is a very good fit to the data, because such a simple model roughly accounts for the vertical scale height as a function of radius. The derived slope of -0.71 is consistent with a "flat" disk geometry that one tends to find for Herbig Be stars (Acke et al. 2008; Kraus et al. 2008a).
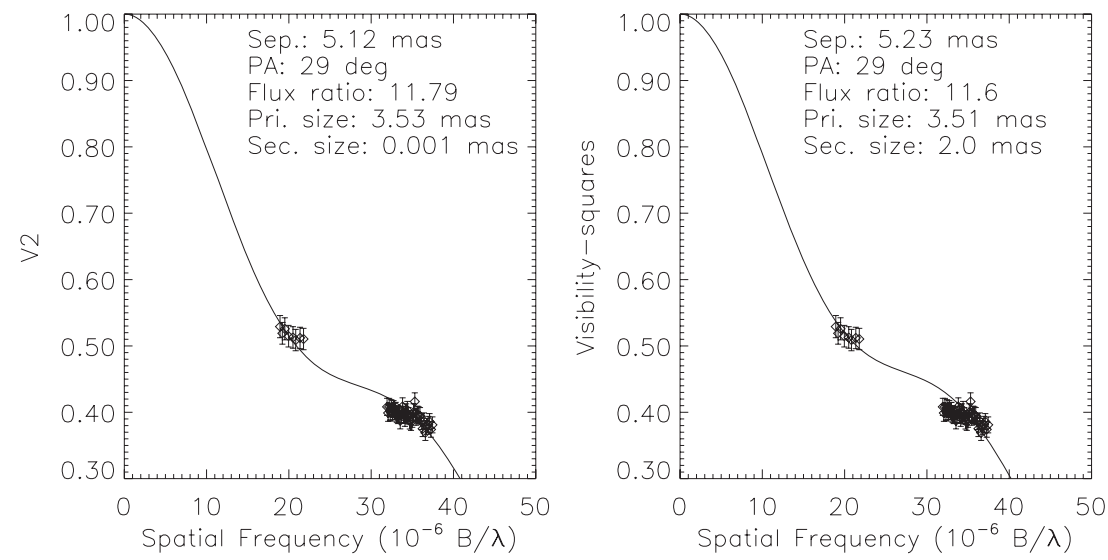

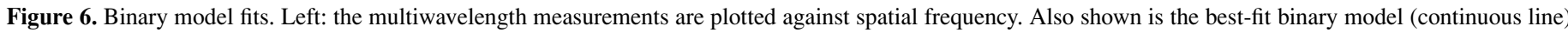
assuming an unresolved companion. Right: same as the previous caption but for a companion of 2 mas diameter. 


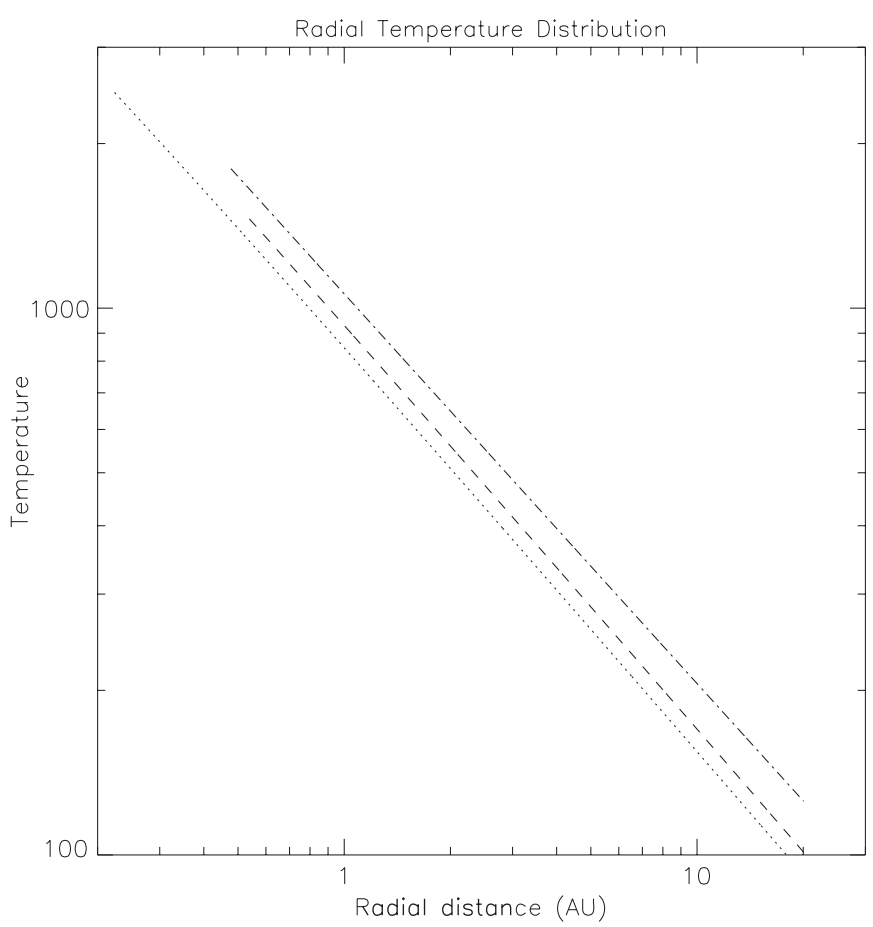

Figure 7. Radial temperature profiles of various disk models. The dotted and dashed lines refer to the two cases of the classical-accretion disk model (Section 3.2; Table 1). The dotted-dashed line refers to the power-law temperature gradient disk model (Section 3.3; Table 1).

Earlier studies (Monnier \& Millan-Gabet 2002; Eisner et al. 2004) using broadband, usually single-wavelength, interferometric data recognized a difference in the near-infrared size versus luminosity behavior of high-luminosity objects (premain sequence $\mathrm{Be}$ ) compared with lower luminosity ones (pre-main sequence Ae), the former being more consistent with "classical disk" models. This has been revisited most recently by Vinković \& Jurkić (2007), who use a modelindependent comparison of visibility to scaled baseline and find a distinction between low-luminosity $\left(\lesssim 10^{3} L_{\odot}\right)$ and high-luminosity $\left(\gtrsim 10^{3} L_{\odot}\right)$ YSO disks where the luminosity break point corresponds to approximate spectral type B3-B5. These authors used a ring and halo model for low-luminosity Herbigs, a halo alone for $\mathrm{T}$ Tauris, and an accretion disk for high-luminosity Herbigs.

However, multiwavelength interferometric studies have not always supported these conclusions when objects are modeled in detail. Kraus et al. (2008a) observed the B6 star MWC 147 in the $H, K$, and $N$ bands. They performed Monte Carlo modeling and found that the interferometric and the SED date were not well fit with a standard, irradiated accretion disk alone, but were well fit with the standard disk plus emission from hot, optically thick gas within the innermost radius of the dust disk. In contrast, Acke et al. (2008) observed the B1.5 star MWC 297 in the $H, K$, and $N$ bands and found that they could not fit the data with a single accretion disk, even with the radial temperature exponent as a free parameter. Instead, they used a three-component geometric model, with characteristic blackbody temperatures of 1700,920 , and $520 \mathrm{~K}$. Our results show that MWC $419\left(\mathrm{~B} 8,330 L_{\odot}\right)$ has the disk characteristics of a high-luminosity object in the categories of Monnier \& Millan-Gabet (2002) but a luminosity lower than the break point of $10^{3} L_{\odot}$ identified by Vinković \& Jurkić (2007). Examining Figure 2 of Vinković \& Jurkić (2007) clearly shows that MWC 419 fits well within the population of lower luminosity Herbigs in their model-independent comparison. Their physical interpretation of the low-luminosity Herbig group is an optically thick disk with an optically thin dust sublimation cavity and an optically thin dusty outflow. Our multiwavelength results do not support grouping MWC 419 in that physical model, but perhaps incorporating multiwavelength data in the model-independent visibility groupings would have produced a different result. The new multiwavelength data set presented here provides significant new information to aid in determining the physical conditions of these young stars, and our data show that the disk surrounding the B8 star MWC 419 is closer in physical characteristics to the more massive Be stars than to the Herbig Ae and T Tauri stars.

Another result from the spectrally dispersed data is that the measured $V^{2}$ is marginally higher at $2.17 \mu \mathrm{m}$ than at adjacent wavelengths. This $V^{2}$ feature is seen in all four independent data sets. As this is the wavelength of $\mathrm{Br} \gamma$, and Brackett recombination emission lines such as $\mathrm{Br} \gamma$ and $\mathrm{Br} 10-20$ are observed in the spectrum of MWC 419 (Harvey 1984), we attribute the difference in line and continuum visibilities to a more compact $\mathrm{Br} \gamma$ emitting region. Similar behavior in the apparent size in the $\mathrm{Br} \gamma$ line compared with the neighboring continuum has been seen before in spectrally resolved interferometry from both VLTI and KI (Kraus et al. 2008b; Eisner et al. 2009). The derived uniform-disk diameter for the $\mathrm{Br} \gamma$ emitting region is $3.33 \pm 0.03$ mas, which is $\sim 4 \%$ less than the continuum size (3.46 mas) around $\mathrm{Br} \gamma$ (Figure 2). The significance level of this detection of the $\mathrm{Br} \gamma$ emitting region with respect to the continuum region is $\sim 2 \sigma$. The actual size of the $\mathrm{Br} \gamma$ emission-line region could be much smaller than the value reported here because of the coarse spectral resolution of our measurements. Detection of $\mathrm{Br} \gamma$ emission inside the innermost dust radius suggests that the disk is optically thin in the inner region where atomic hydrogen gas exists.

\section{SUMMARY}

This article reports the first milliarcsecond angular resolution observations of a HAeBe star (MWC 419) providing $L$-band, as well as simultaneously obtained $K$-band data, both spectrally dispersed. This multiwavelength observational capability is well suited to probing the temperature distribution in the inner regions of YSO disks, which is very important for distinguishing between models and gaining insight into the three dimensional geometry of the inner disk. Such measurements could distinguish discrete spatial distributions, such as dust rims, from relatively smooth spatial distributions, such as classical accretion disks, based on their distinct wavelength dependent disk sizes. In addition, interferometric measurements in the relatively unexplored $L$-band provide needed constraints to the disk/envelope geometry and temperature structure.

Simple geometrical pole-on disk models are used to infer a linear relationship between the derived object size and wavelength in the $2-4 \mu \mathrm{m}$ region, suggesting a simple physical model for the disk. The steep slopes of these linear relations imply that the disk is extended with a radial temperature gradient. We find that the accretion disk model of Hillenbrand et al. (1992) derived from SED analysis does not fit our interferometric measurements. An updated accretion disk model with accretion rate 1.5 times larger and inner cavity 2.4 times larger fits the $K$ band data well, but lies $3 \sigma$ above the $L$ band data. However, both of these classical accretion disk models predict an unrealistically large accretion rate of $\sim 3 \times 10^{-5} M_{\odot} \mathrm{yr}^{-1}$ to fit the SED data. A power-law temperature profile with a slightly 
shallower slope of -0.71 fits both the spectrally dispersed interferometric measurements and the SED satisfactorily, suggesting a relatively flat disk geometry for MWC 419. The measured disk size at $\mathrm{Br} \gamma$ reveals the presence of compact emitting hydrogen gas in the inner regions of the disk. A more complete sample of YSO disk observations with adequate wavelength and $(u, v)$ coverage, plus a detailed radiative transfer modeling, is required to address the intriguing inner disk geometry in these sources.

Keck Interferometer is funded by the National Aeronautics and Space Administration (NASA). Observations presented were obtained at the W. M. Keck Observatory, which is operated as a scientific partnership among the California Institute of Technology, the University of California, and NASA. The Observatory was made possible by the generous financial support of the W. M. Keck Foundation. We thank E. Appleby, B. Berkey, A. Booth, A. Cooper, S. Crawford, W. Dahl, C. Felizardo, J. Garcia-Gathright, J. Herstein, R. Ligon, D. Medeiros, D. Morrison, T. Panteleeva, B. Parvin, B. Smith, K. Summers, K. Tsubota, C. Tyau, and E. Wetherell for their contributions to the instrument development, integration, and operations. S. Ragland also thanks M. Hrynevych, M. Kassis, and J. Woillez for useful discussions. We thank the referee for constructive critical comments that helped us to significantly improve the paper.

\section{REFERENCES}

Acke, B., et al. 2008, A\&A, 485, 209

Akeson, R. L., et al. 2005, ApJ, 635, 1173

Bernacca, P. L., et al. 1993, A\&A, 278, 47

Berrilli, F., Corciulo, G., Ingrosso, G., Lorenzetti, D., Nisini, B., \& Strafella, F. 1992, ApJ, 398, 254

Boehm, T., \& Catala, C. 1995, A\&A, 301, 155

Bouret, J.-C., \& Catala, C. 1998, A\&A, 340, 163

Brooke, T. Y., Tokunaga, A. T., \& Strom, S. E. 1993, AJ, 106, 656

Cardelli, J. A., Clayton, G. C., \& Mathis, J. S. 1989, ApJ, 345, 245

Chen, P. S., Wang, X. H., \& He, J. H. 2000, Ap\&SS, 271, 259
Colavita, M., et al. 1999, ApJ, 510, 505

Colavita, M., et al. 2003, ApJ, 592, 83

Dullemond, C. P., Dominik, C., \& Natta, A. 2001, ApJ, 560, 957

Eisner, J. A., Lane, B. F., Akeson, R. L., Hillenbrand, L. A., \& Sargent, A. I. 2003, ApJ, 588, 360

Eisner, J. A., Lane, B. F., Hillenbrand, L. A., Akeson, R. L., \& Sargent, A. I. 2004, ApJ, 613, 1049

Eisner, J. A., Chiang, E. I., Lane, B. F., \& Akeson, R. L. 2007, ApJ, 657, 347

Eisner, J. A., Graham, J. R., Akeson, R. L., \& Najita, J. 2009, ApJ, 692, 309

Fuente, A., Martin-Pintado, J., Bachiller, R., Rodriguez-Franco, A., \& Palla, F. 2002, A\&A, 387, 977

Hartmann, L., Kenyon, S. J., \& Calvet, N. 1993, ApJ, 407, 219

Harvey, P. M. 1984, PASP, 96, 297

Herbig, G. H. 1960, ApJS, 4, 337

Hillenbrand, L. A. 1995, PhD thesis, Univ. of Massachusetts

Hillenbrand, L. A., Strom, S. E., Vrba, F. J., \& Keene, J. 1992, ApJ, 397, 613

Isella, A., \& Natta, A. 2005, A\&A, 438, 899

Isella, A., Tatulli, E., Natta, A., \& Testi, L. 2008, A\&A, 483, L13

Kraus, S., Preibisch, T., \& Ohnaka, K. 2008a, ApJ, 676, 490

Kraus, S., et al. 2008b, A\&A, 489, 1157

Kurchakov, A. V., Pogodin, M. A., \& Rspaev, F. K. 2007, Astrophysics, 50, 207

Lattanzi, M. G., Massone, G., \& Munari, U. 1991, AJ, 102, 177

Marston, A. P., \& McCollum, B. 2008, A\&A, 477, 193

Millan-Gabet, R., Malbet, F., Akeson, R., Leinert, C., Monnier, J., \& Waters, R. 2007, in Protostars and Planets V, ed. B. Reipurth, D. Jewitt, \& K. Keil (Tucson, AZ: Univ. of Arizona Press), 539

Millan-Gabet, R., Schloerb, F. P., \& Traub, W. A. 2001, ApJ, 546, 358

Miroshnichenko, A., Ivezic, Z., \& Elitzur, M. 1997, ApJ, 475, L41

Monnier, J. D., \& Millan-Gabet, R. 2002, ApJ, 579, 694

Monnier, J. D., et al. 2005, ApJ, 624, 832

Natta, A., Prusti, T., Neri, R., Wooden, D., Grinin, V. P., \& Mannings, V. 2001, A\&A, 371, 186

Pezzuto, S., Strafella, F., \& Lorenzetti, D. 1997, ApJ, 485, 290

Pirzkal, N., Spillar, E. J., \& Dyck, H. M. 1997, ApJ, 481, 392

Ragland, S., et al. 2008, Proc. SPIE, 7013, 10

Skinner, S. L., Brown, A., \& Stewart, R. T. 1993, ApJS, 87, 217

Subramaniam, A., Mathew, B., \& Kartha, S. S. 2006, Bull. Astron. Soc. India, 34,315

Tannirkulam, A., et al. 2008, ApJ, 689, 513

Tuthill, P. G., Monnier, J. D., \& Danchi, W. C. 2001, Nature, 409, 1012

van Belle, G. T. 1999, PASP, 111, 1515

van den Ancker, M. E., de Winter, D., \& Tjin A Djie, H. R. E. 1998, A\&A, 330, 145

Vinković, D., \& Jurkić, T. 2007, ApJ, 658, 462

Wilkin, F. P., \& Akeson, R. L. 2003, Ap\&SS, 286, 145 